\title{
Epithelial-mesenchymal transition-related microRNA-200s regulate molecular targets and pathways in renal cell carcinoma
}

\author{
Hirofumi Yoshino ${ }^{1,4}$, Hideki Enokida ${ }^{1,4}$, Toshihiko Itesako ${ }^{1}$, Shuichi Tatarano ${ }^{1}$, Takashi Kinoshita ${ }^{2}$, Miki Fuse ${ }^{2}$, \\ Satoko Kojima ${ }^{3}$, Masayuki Nakagawa ${ }^{1}$ and Naohiko Seki ${ }^{2}$
}

Our recent studies of microRNA (miRNA) expression signatures demonstrated that the epithelial-mesenchymal transition (EMT)-related microRNA-200 family (miR-200s: $m i R-200 a / b / c, m i R-141$ and $m i R-429$ ) were significantly downregulated in renal cell carcinoma (RCC) and putative tumor-suppressive miRNAs in RCC. In this study, our aim was to investigate the functional significance of the miR-200s in cancer cells and to identify novel miR-200s-regulated molecular targets and pathways in RCC. Expression levels of all the miR-200s members were significantly downregulated in human RCC tissues compared with normal renal tissues. Restoration of mature miR-200s in RCC cell line resulted in significant inhibition of cell proliferation and migration, suggesting that miR-200s function as tumor suppressors in RCC. Furthermore, we utilized gene expression analysis and in silico database analysis to identify miR-200s-regulated molecular targets and pathways in RCC. The miR-200s was categorized into two groups, according to their seed sequences, $m i R-200 \mathrm{~b} / \mathrm{c} / 429$ and $m i R-200 \mathrm{a} / 141$. Our data demonstrated that the 'Focal adhesion' and 'ErbB signaling' pathways were significantly regulated by miR-200b/c/429 and $m i R-200 a / 141$, respectively. The identification of novel tumor-suppressive miR-200s-regulated molecular targets and pathways has provided new insights into RCC oncogenesis and metastasis.

Journal of Human Genetics (2013) 58, 508-516; doi:10.1038/jhg.2013.31; published online 2 May 2013

Keywords: miR-200a; miR-200b; miR-200c; miR-141; miR-429; renal cell carcinoma; tumor suppressor

\section{INTRODUCTION}

Renal cell carcinoma (RCC) is the most common neoplasm of the adult kidney, and the incidence and mortality rates of RCC are increasing at a rate of $2-3 \%$ per decade. ${ }^{1}$ Although surgery is often curative for localized disease, a significant percentage of these patients develop relapses or metastatic disease, which are associated with poor prognosis. The 5-year survival rate of advanced stage of RCC is very poor $(5-10 \%)$ because of recurrence or distant metastasis. ${ }^{2}$ To improve treatment of RCC, targeted molecular therapies that have been developed are currently being widely used for patients with metastatic or recurrent RCC. However, these targeted therapies are insufficient for patients who have relapsed or have metastatic disease. ${ }^{3}$ Therefore, it is important to understand the molecular mechanisms underlying RCC recurrence and metastasis, as this knowledge may be used to help improve RCC treatment.

The discovery of non-coding RNAs in the human genome was an important conceptual breakthrough in the post-genome sequencing era. ${ }^{4}$ Improved understanding of non-coding RNAs is necessary for continued progress in cancer research. MicroRNAs (miRNAs) are a class of small non-coding RNA molecules of 19-22 nucleotides that regulate protein-coding gene expression by repressing translation or cleaving RNA transcripts in a sequence-specific manner. ${ }^{5}$ A growing body of evidence suggests that miRNAs are aberrantly expressed in several types of human cancer, and that they have significant roles in the initiation, progression and metastasis of human cancers. ${ }^{6}$

It is believed that normal regulatory mechanisms can be disrupted by aberrant expression of tumor-suppressive or oncogenic miRNAs in cancer cells. Thus, identification of aberrantly expressed miRNAs is an important step in elucidating miRNA-mediated oncogenic pathways.

On the basis of recent reports describing miRNA signatures, several downregulated miRNAs have been identified. Here, we analyzed the miRNA expression signatures published in the literature to identify miRNA candidates involved in RCC. ${ }^{7-16}$ We chose to focus on the $m i R-200 s(m i R-200 a / b / c, m i R-141$ and $m i R-429)$, which is frequently downregulated in clinical specimens from RCC patients.

Our recent studies of miRNA expression signatures also demonstrated that the epithelial-mesenchymal transition (EMT)-related miR-200s members were significantly downregulated in RCC. ${ }^{8}$

${ }^{1}$ Department of Urology, Graduate School of Medical and Dental Sciences, Kagoshima University, Kagoshima, Japan; ${ }^{2}$ Department of Functional Genomics, Chiba University Graduate School of Medicine, Chiba, Japan and ${ }^{3}$ Department of Urology, Teikyo University Chiba Medical Center, Chiba, Japan

${ }^{4}$ These two authors contributed equally to this work.

Correspondence: Dr N Seki, Department of Functional Genomics, Chiba University Graduate School of Medicine, 1-8-1 Inohana Chuo-ku, Chiba 260-8670, Japan. E-mail: naoseki@faculty.chiba-u.jp

Received 3 March 2013; revised 2 April 2013; accepted 3 April 2013; published online 2 May 2013 
The initial steps of invasion and metastasis involve cellular changes consistent with EMT, which is associated with a loss of epithelial characteristics; such as, the intercellular adhesion protein E-cadherin and an apical-basal polarity, and an acquisition of mesenchymal markers; such as, vimentin, collagen, fibronectin, and the E-cadherin transcriptional repressors; such as ZEB1 and ZEB2. ${ }^{17,18}$ The miR-200s has been established as an important regulator of tumor invasion and metastasis through its targeting of ZEB1 and ZEB2. ${ }^{19}$ To date, molecular pathways regulated by the $m i R-200$ s has not been fully investigated in RCC. Greater understanding of the molecular pathways regulated by the $m i R-200$ s could lead to the development of better prognostic, diagnostic and therapeutic interventions for RCC.

The aim of this study was to investigate the functional significance of the miR-200s members and to identify the molecular targets and pathways regulated by the miR-200s members in RCC cells. The results here identified several cancer-related genes and pathways, including 'Focal adhesion' and 'ErbB signaling' pathways, as targets of the miR-200s members. These novel miR-200s-regulated signaling pathways and targeted genes provide new insights into the mechanisms of RCC oncogenesis and suggest novel therapeutic strategies for treatment of this disease.

\section{MATERIALS AND METHODS}

\section{Selection of aberrantly expressed miRNAs by RCC miRNA} expression signatures

We searched PubMed for publications on RCC miRNA signatures using the keywords renal cell carcinoma, kidney cancer and miRNA on 23 July 2012. We selected articles in English in which the scientific detail and reporting were sufficient to enable our understanding and that presented novel findings. Our previous miRNA expression signature study was included in the selected articles. $^{8}$ We hypothesized that frequently observed low expression of miRNAs are involved in the progression and metastasis of RCC. On the basis of this point, we quantified the number of times that specific downregulated miRNAs appeared in expression signatures.

\section{Clinical specimens and cell culture}

A total of 29 clear-cell RCC and 27 normal kidney specimens were collected from patients who had undergone radical nephrectomies at Kagoshima University Hospital. Patient characteristics and representative pathological features are shown in Supplementary Table 1. The samples were processed and stored in RNAlater (Qiagen, Valencia, CA, USA) at $4{ }^{\circ} \mathrm{C}$ until RNA extraction. These specimens were staged according to the American Joint Committee on Cancer-Union Internationale Contre le Cancer tumour-node-metastasis classification and histologically graded. ${ }^{20}$ This study was approved by the Bioethics Committee of Kagoshima University, with written prior informed consent and approval given by the patients.

\section{Cell culture and RNA extraction}

Human RCC cell line, Caki-1, obtained from the American Type Culture Collection (Manassas, VA, USA), was derived from skin metastasis. Therefore, the results from analyses might be more plausible to understand the EMT-related genes and pathways than using other cell lines. The cell line was incubated in RPMI-1640 medium with $10 \%$ fetal bovine serum and maintained in a humidified incubator $\left(5 \% \mathrm{CO}_{2}\right)$ at $37^{\circ} \mathrm{C}$. Total RNA was extracted, as described previously. ${ }^{8}$

\section{Quantitative real-time RT-PCR}

TaqMan probes and primers for ZEB1 (P/N: Hs00232783_ml; Applied Biosystems, Foster City, CA, USA), ZEB2 (P/N: Hs00207691_m1; Applied Biosystems) and CDH1 (E-cadherin, P/N: Hs01023894_ml; Applied Biosystems) were assay-on-demand gene expression products. All reactions were performed in duplicate and a negative control lacking cDNA was included. We followed the manufacturer's protocol for polymerase chain reaction (PCR) conditions. Stem-loop reverse transcription (RT)-PCR (TaqMan MiRNA Assays; Assay ID: 000502 for miR-200a, 002251 for miR-200b, 002300 for $m i R-200 c, 000463$ for $m i R-141$ and 001024 for miR-429; Applied Biosystems) was used to quantify miRNAs according to previously published conditions. ${ }^{21}$ To normalize the data for quantification of the miRNAs, we used human RNU48 (P/N: 001006; Applied Biosystems), and the delta-delta Ct method was used to calculate the fold-change.

\section{Mature miRNA transfection}

As described elsewhere, ${ }^{21}$ the RCC cell lines were transfected with Lipofectamine RNAiMAX transfection reagent (Invitrogen, Carlsbad, CA, USA) and Opti-MEM (Invitrogen) with $10 \mathrm{~nm}$ mature miRNA molecules (hsa-miR-200a for PM10991, hsa-miR-200b for PM10492, hsa-miR-200c for PM11714, hsa-miR-141 for PM10860 and hsa-miR-429 for PM10221). Negative control miRNA (CN-002000-01-05; Thermo Fisher Scientific, Waltham, MA, USA) were used in the gain-of-function experiments. We evaluated the transfection efficiency of the miRNAs in RCC cell lines based on the downregulation of mRNA expression levels of protein tyrosine kinase 9 (alias twinfilin: TWF1) after miR-1 transfection as described previously. ${ }^{22}$ Cells were seeded in six-well plates for wound-healing assays $\left(20 \times 10^{4}\right.$ cells per well) and in 96-well plates for XTT (2,3-bis(2-methoxy-4nitro-5-sulfophenyl)-2H-tetrazolium-5-carboxanilide)) assays (3000 per well).

\section{Cell proliferation and migration assays}

Cell proliferation was determined with an XTT assay according to the manufacturer's instructions (Roche Applied Science, Tokyo, Japan) $72 \mathrm{~h}$ after transfection. Cell migration activity was evaluated with a wound-healing assay. Cells were seeded in six-well dishes, and the cell monolayer was scraped using a P-20 micropipette tip. The initial gap length $(0 \mathrm{~h})$ and the residual gap length $24 \mathrm{~h}$ after wounding were calculated from photomicrographs. All experiments were performed in triplicate.

Identification of significantly enriched signaling pathways of the miR-200s members using oligo-microarray and in silico analysis

Oligo-microarray Human 44K (Agilent Technologies, Santa Clara, CA, USA) was used for expression profiling in each miR-200s family-transfected Caki-1 cell in comparison with miR-negative control transfectants, as described previously. ${ }^{23}$ Briefly, the hybridization and washing steps were performed in accordance with the manufacturer's instructions. The arrays were scanned using a Packard GSI Lumonics ScanArray 4000 (Perkin-Elmer, Boston, MA, USA). The data obtained were analyzed with the DNASIS array software (Hitachi Software Engineering, Tokyo, Japan), which converted the signal intensity for each spot into text format. The $\log 2$ ratios of the median subtracted background intensity were analyzed. Data from each microarray study were normalized by global normalization.

Their predicted target genes and their miRNA binding site seed regions were investigated using TargetScan (http://www.targetscan.org/). The sequences of the predicted mature miRNAs were confirmed using miRBase (release 19.0, August 2012; http://www.mirbase.org/).

To identify signaling pathways regulated by the miR-200 family in RCC, upregulated genes that were targeted by these miRNAs were analyzed in the Kyoto Encyclopedia of Genes and Genomes (KEGG) pathway categories using the GENECODIS program (http://genecodis.cnb.csic.es/).

\section{Statistical analysis}

The relationships between two variables and the numerical values obtained by real-time RT-PCR were analyzed using the Mann-Whitney $U$-test. The relationships among three variables and the numerical values were analyzed using the Bonferroni-adjusted Mann-Whitney $U$-test. Spearman's rank test was used to evaluate the relationships among the relative expression levels of the miR-200s family. All statistical analyses were performed using the Expert StatView analysis software (version 4; SAS Institute Inc., Cary, NC, USA). In the comparison among six variables, a non-adjusted statistical level of significance of $P<0.05$ corresponds to a Bonferroni-adjusted level of $P<0.0033$ 


\section{RESULTS}

Aberrantly expressed miRNAs based on the RCC expression signatures In this study, we listed 10 miRNA expression signatures of RCC based on results of database search. ${ }^{7-16}$ We quantified the number of times that specific downregulated miRNAs appeared (Supplementary Table 2 and summarized in Table 1). Detailed information about miRNA expression signatures; such as, sample numbers and the result of each study, was shown in Supplementary Table 2. We recognized that $m i R-200 b, m i R-200 c, m i R-141$ and $m i R-429$ were the most frequently downregulated miRNAs (Table 1 ), and miR-200a was also downregulated in RCC signatures (5 of 10 signatures). Thus, we focused on the miR-200s members and investigated the functional significance and molecular pathways regulated by the miR-200s.

The miR-200s members are clustered on two different chromosomal regions; miR-200b, miR-200a and miR-429 are located on chromosome $1 \mathrm{p} 36.33$, whereas $m i R-200 c$ and $m i R-141$ are on the chromosome 12 p13.31 (Figure 1Aa). The miR-200s can also be classified, according to their seed sequence arrangement, into two groups: $m i R-200 b / c / 429$ and $m i R-200 a / 141$ (Figure 1Ab).

Expression levels of $m i R-200 s$ in clinical RCC specimens and the Caki-1 RCC cell line

We evaluated expression levels of the miR-200s members in clinical RCCs $(n=29)$, in normal specimens $(n=27)$ and in the Caki-1 RCC cell line. Quantitative stem-loop RT-PCR demonstrated that the expression levels of $m i R-200 \mathrm{~b} / \mathrm{miR}-200 \mathrm{a} / \mathrm{miR}-429$ were significantly lower in clinical RCC specimens and in Caki-1 compared with pathologically normal tissues (each, $P<0.0001$; Figure $1 \mathrm{~B}$, upper). Furthermore, there were significant positive correlations between the expression levels of those clustered miRNAs (each, $P<0.0001$; Figure $1 \mathrm{~B}$, lower). The same results were obtained for $m i R-200 c$ and miR-141 $(P<0.0001$, each; Figure 1C).

There was no significant relationship between the clinicopathological parameters and the expression levels of the miR-200s in this study (data not shown).

\section{EMT-related gene expression in miR-200s-transfected Caki-1 RCC cell line}

To select RCC cell lines suitable for the analysis, we evaluated the mRNA expression levels of EMT-associated genes, ZEB1, ZEB2 and $C D H 1$, in miR-200s-transfected RCC cell lines (Caki-1, Caki-2 and ACHN). Quantitative real-time RT-PCR analysis showed that the mRNA expression levels of $Z E B 1$ and $Z E B 2$ were significantly downregulated in Caki-1 cells compared with control $(P<0.0001$, each; Figure 2a). In addition, the mRNA expression levels of $C D H 1$ were significantly upregulated in $m i R-200 b, m i R-200 c$ and $m i R-429$ transfected cells compared with the miR-control transfectant $(P<0.0001$, each). There were trends but no significant differences in $\mathrm{CDH} 1$ mRNA expression in the miR-200a and miR-141-transfected Caki-1

Table 1 Consistently reported downregulated miRNAs in profiling studies

\begin{tabular}{|c|c|c|c|c|c|c|}
\hline \multirow{2}{*}{$\begin{array}{l}\text { No. of } \\
\text { studies }\end{array}$} & \multirow{2}{*}{$\begin{array}{l}\text { hsa-mature } \\
\text { sequence }\end{array}$} & \multirow{2}{*}{$\begin{array}{l}\text { Stem-loop } \\
\text { sequence }\end{array}$} & \multirow[b]{2}{*}{ Locus } & \multicolumn{2}{|c|}{ Host genes } & \multirow[b]{2}{*}{ Clustered miRNAs } \\
\hline & & & & Sense & Antisense & \\
\hline 9 & hsa-miR-200c & hsa-miR-200c & $12 \mathrm{p} 13$ & & \multirow{13}{*}{ RP11 } & hsa-miR-141 \\
\hline 8 & hsa-miR-141 & hsa-miR-141 & $12 \mathrm{p} 13$ & U47924.27-001 & & hsa-miR-200c \\
\hline 8 & hsa-miR-429 & hsa-miR-429 & $1 p 36$ & & & hsa-miR-200b/hsa-miR-200a \\
\hline 7 & hsa-miR-200b & hsa-miR-200b & $1 p 36$ & & & hsa-miR-200a/hsa-miR-429 \\
\hline 7 & hsa-miR-514a-3p & hsa-miR-514a-1 & Xq27 & & & hsa-miR-514a-3/hsa-miR-514a-2/hsa-miR-510 \\
\hline 6 & hsa-miR-204 & hsa-miR-204 & $9 q 21$ & TRPM3 & & - \\
\hline \multirow[t]{2}{*}{6} & hsa-miR-218 & hsa-miR-218-1 & $4 p 15$ & SLIT2 & & - \\
\hline & & hsa-miR-218-2 & $5 q 34$ & SLIT3 & & - \\
\hline 5 & hsa-miR-30a-5p & hsa-miR-30 & $6 q 13$ & \multirow[t]{5}{*}{ C6orf155/LINC00472 } & & - \\
\hline 5 & hsa-miR-133b & hsa-miR-133b & $6 p 12$ & & & hsa-miR-206 \\
\hline 5 & hsa-miR-138 & hsa-miR-138-2 & $16 q 13$ & & & - \\
\hline 5 & hsa-miR-200a & hsa-miR-200a & $1 p 36$ & & & hsa-miR-200b/hsa-miR-429 \\
\hline 5 & hsa-miR-363 & hsa-miR-363 & & & & $\begin{array}{l}\text { hsa-miR-106a/hsa-miR-18b/hsa-miR-20b/hsa-miR-19b-2/hsa-miR- } \\
\text { 19a-2 }\end{array}$ \\
\hline 4 & hsa-miR-30a-3p & hsa-miR-30 & $6 q 13$ & \multirow{2}{*}{\multicolumn{2}{|c|}{ C6orf155/LINC00472 }} & 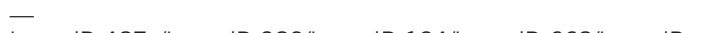 \\
\hline 4 & hsa-miR-154 & hsa-miR-154 & $14 q 32$ & & & $\begin{array}{l}\text { hsa-miR-487a/hsa-miR-382/hsa-miR-134/hsa-miR-668/hsa-miR- } \\
\text { 485/a-hsa-miR-323b/hsa-miR-496/hsa-miR-377/hsa-miR-541/hsa- } \\
\text { miR-409/hsa-miR-412/hsa-miR-369/hsa-miR-410/hsa-miR-656 }\end{array}$ \\
\hline 4 & hsa-miR-187 & hsa-miR-187 & $18 q 12$ & & & - \\
\hline 4 & hsa-miR-335 & hsa-miR-335 & 7q32 & \multirow{2}{*}{\multicolumn{2}{|c|}{ MEST }} & - \\
\hline 4 & hsa-miR-510 & hsa-miR-510 & Xq27 & & & hsa-miR-514a-2/hsa-miR-514a-1 \\
\hline 4 & hsa-miR-532-5p & hsa-miR-532 & Xp11 & \multicolumn{2}{|l|}{ CLCN5 } & $\begin{array}{l}\text { hsa-miR-188/hsa-miR-500a/hsa-miR-362/hsa-miR-50.1/hsa-miR- } \\
\text { 500b }\end{array}$ \\
\hline 3 & hsa-miR-100 & hsa-miR-100 & $11 q 24$ & & & hsa-let-7a-2 \\
\hline 3 & hsa-miR-135a & hsa-miR-135-1 & $3 q 21$ & RP11/GLYCTK-AS1 & \multirow{2}{*}{$\begin{array}{l}\text { GLYCTK } \\
\text { RP11 }\end{array}$} & - \\
\hline 3 & hsa-miR-135b & $\begin{array}{l}\text { hsa-miR-13b-2 } \\
\text { hsa-miR-135b }\end{array}$ & $\begin{array}{l}12 \mathrm{q} 23 \\
1 \mathrm{q} 32\end{array}$ & LEMD1 & & - \\
\hline 3 & hsa-miR-136 & hsa-miR-136 & $14 q 32$ & HSA-MIR136 & RTL1 & $\begin{array}{l}\text { hsa-miR-665/hsa-miR-431/hsa-miR-433/hsa-miR-127/hsa-miR- } \\
432\end{array}$ \\
\hline 3 & hsa-miR-149 & hsa-miR-149 & $2 q 37$ & GPC1 & AC1 10619.2 & - \\
\hline 3 & hsa-miR-184 & hsa-miR-184 & $15 q 25$ & & RP11 & - \\
\hline 3 & hsa-miR-199a-5p & hsa-miR-199a-1 & $19 p 13$ & & DNM2 & - \\
\hline 3 & hsa-miR-362-5p & hsa-miR-362 & Xp11 & CLCN5 & & $\begin{array}{l}\text { hsa-miR-532/hsa-miR-188/hsa-miR-500a/hsa-miR-501/hsa-miR- } \\
\text { 500b/hsa-miR-660/hsa-miR-502 }\end{array}$ \\
\hline 3 & hsa-miR-411 & hsa-miR-411 & $14 q 32$ & & & $\begin{array}{l}\text { hsa-miR-379/hsa-miR-299/hsa-miR-380/hsa-miR-1197/hsa-miR- } \\
\text { 323a/hsa-miR-758/hsa-miR-329-1/hsa-miR-329-2/hsa-miR-494/ } \\
\text { hsa-miR-1193/hsa-miR-543 }\end{array}$ \\
\hline 3 & hsa-miR-507 & hsa-miR-507 & Xq27 & & & hsa-miR-508/hsa-miR-506/hsa-miR-513a-2 \\
\hline 3 & hsa-miR-532-3p & hsa-miR-532 & Xp11 & CLCN5 & & $\begin{array}{l}\text { hsa-miR-188/hsa-miR-500a/hsa-miR-362/hsa-miR-501/hsa-miR- } \\
\text { 500b }\end{array}$ \\
\hline
\end{tabular}

Abbreviation: miR, microRNA. 
Human Chromosome 1p36.33

1102000

1105000

$\longleftrightarrow$ miR-200b miR-200a

Human Chromosome 12p13.31

7072000

7075000

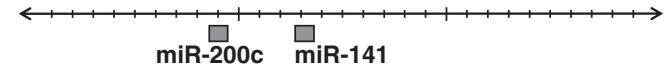

b
hsa-miR-200b
5' UAAUACUgCCUgGUAAUgAUgA $3^{\prime}$
hsa-miR-200c
5' UAAUACUgCCUgGUaAUgaUgGa $3^{\prime}$
hsa-miR-429
5' UAAUACUGUCUGGUAAAACCGU 3'
hsa-miR-200a
5' UAACACUGUCUGGUAACGAUGU $3^{\prime}$
hsa-miR-141
5' UAACACUgUCUgGUAAAGAUgG 3 '

B
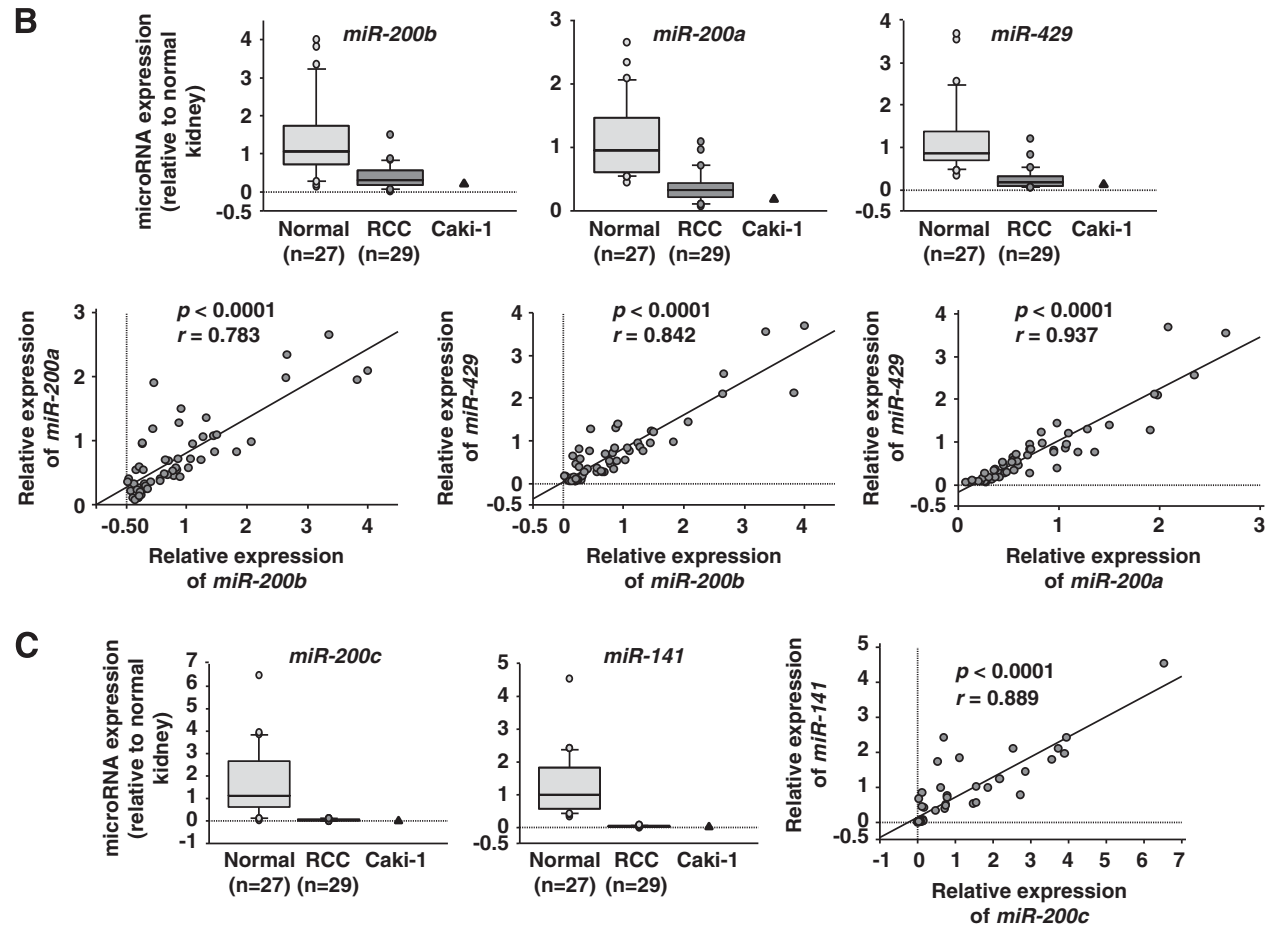

Figure 1 Chromosomal locations and sequence alignment of miR-200s members in the human genome and their expression levels in RCCs. (Aa) MiR200b, miR-200a and miR-429 locations on chromosome 1, and miR-200c and miR-141 locations on chromosome 12. (Ab) The miR-200 family consists of two closely related subfamilies defined by their seed sequences. Nucleotides $2-8$, representing the seed sequence, are underlined. (B, upper) Expression levels of miR-200b, miR-200a and miR-429 in clinical RCC specimens and in Caki-1 compared with clinical normal tissues. (B, lower) Significant positive correlation is recognized between the expression levels of miR-200b, miR-200a and miR-429. (C, left) Expression levels of miR-200c and miR-141 in clinical RCC specimens and in Caki-1 compared with clinical normal tissues. (C, right) Significant positive correlation between the expression levels of miR-200c and miR-141.

cells $(P=0.0047$ and 0.0099 , respectively). Other cell lines (Caki-2 and $\mathrm{ACHN}$ ) were not suitable for the analysis because irregular expression of EMT-related genes were detected in those cell lines (data not shown).

\section{Effect of $m i R-200 s$ restoration on Caki-1 cell proliferation and migration}

We performed gain-of-function studies using a Caki-1 cell line that had been transfected with mature miR-200s. The XTT assay demonstrated that cell proliferation was significantly suppressed in each miR-200s transfectant compared with the miR-control transfectant $(P<0.0001$ each; Figure 2b, upper). The wound-healing assay demonstrated that significant inhibition of cell migration occurred in each $m i R$-200s member transfectant, except for $m i R$-200a-transfected cells $(P<0.001$ each; Figure $2 b$, lower). There was a trend but no significant difference in the miR-200a-transfected Caki-1 cell $(P=0.043)$. These results suggest that the $m i R-200$ s has an important role in tumor suppression by downregulating oncogenic genes.

\section{Identification of $m i R-200 s$-regulated target genes and molecular} pathways in RCC

We performed oligo-microarray analysis and in silico analysis (Figure 3). We first performed oligo-microarray using miR-200stransfected Caki-1 cells and found a total of 1438 downregulated 

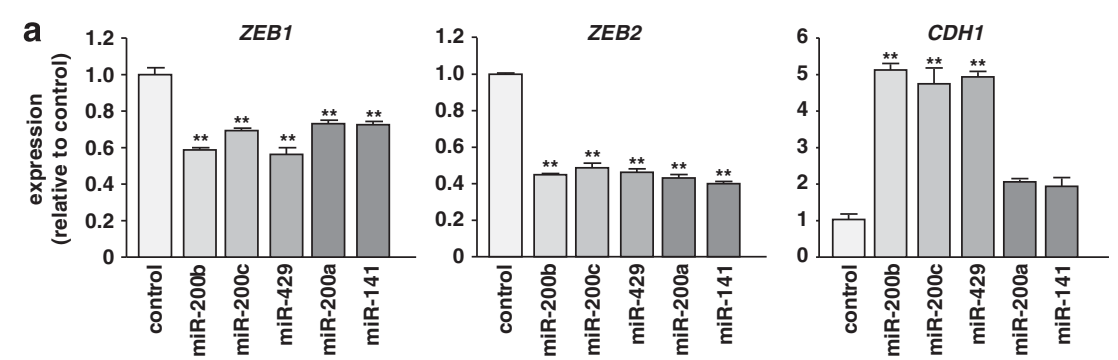

b
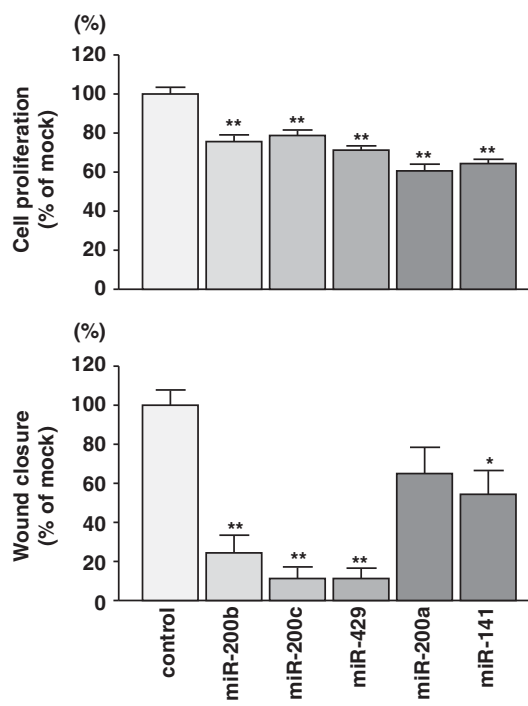

Figure 2 EMT-related gene expression, and the effect of restoring miR-200s in Caki-1 RCC cell line. (a) ZEB1, ZEB2 and CDH-1 mRNA expression after $24 \mathrm{~h}$ transfection with $10 \mathrm{~nm}$ each miR-200 family members and miR-control. (b, upper) Cell proliferation of Caki-1 is determined by XTT assay after $72 \mathrm{~h}$ transfection with $10 \mathrm{~nm}$ of each miR-200 family member and miR-control. (b, lower) Cell migration, as determined by the wound-healing assay. Data showed significant inhibition of cell migration in miR-200b, miR-200c, miR-429 and miR-141 transfectants compared with control. There was a trend in inhibition of cell migration by the miR-200a-transfected Caki-1 cell, but there was no significant difference. ${ }^{*} P<0.001$ and ${ }^{* *} P<0.0001$.

genes in $\mathrm{miR}-200 \mathrm{~b} / \mathrm{miR}-200 \mathrm{c} / \mathrm{miR}$-429-transfected cells and 1050 downregulated genes in $m i R-200 a / m i R-141$-transfected cells compared with miR-control transfectants. Among the genes above, TargetScan database implied that 689 and 451 genes had predicted binding sites for $m i R-200 b / m i R-200 c / m i R-429$ and $m i R-200 a / m i R-141$, respectively.

To investigate the expression status of these genes in RCC clinical specimens, we examined gene expression profiles in the GEO database (accession numbers: GSE36895 and GSE22541). Among the candidate genes, 186 genes identified in $m i R-200 b / m i R-200 c / m i R-429$ transfection and 108 genes identified in $m i R-200 a / m i R-141$ transfection were actually upregulated in 53 RCC specimens compared with 23 noncancerous kidney tissues (Tables $2 \mathrm{a}$ and $\mathrm{b}$ and Supplementary Tables $3 \mathrm{a}$ and $\mathrm{b}$ ). These genes were assigned KEGG annotations by the GENECODIS program, resulting in 28 significantly enriched signaling pathways for $m i R-200 \mathrm{~b} / \mathrm{miR}-200 \mathrm{c} / \mathrm{miR}-429$ and 23 significantly enriched signaling pathways for $m i R-200 a / m i R-141$ (Tables $3 \mathrm{a}$ and $\mathrm{b}$, respectively, $P<0.0001$ ).

\section{DISCUSSION}

In this study, we analyzed aberrantly expressed miRNAs in RCC, based on published data on miRNA expression signatures, including those from our recently published miRNA signature studies. ${ }^{8}$ Members of the EMT-related miR-200s have been frequently observed to be downregulated in RCC signatures, suggesting that these miRNAs function as tumor suppressors in RCC. The miR-200s members form clustered miRNAs in two different human chromosome regions; $m i R-200 b, m i R-200 a$ and $m i R-429$ are located on chromosome $1 \mathrm{p} 36.33$, whereas $m i R-200 c$ and $m i R-141$ are on the chromosome $12 \mathrm{p} 13$. A previous study demonstrated that loss of 1 p36 was observed in $14 \%$ of clear-cell RCCs, whereas gain of 12 p 13.31 was in $19 \%$ of them. ${ }^{24}$ Elucidation of the molecular mechanisms of the silencing of miR-200s expression in RCC cells will be necessary in future.

Downregulation of the miR-200s members has been reported in several cancers; such as, those of breast, liver and ovary. ${ }^{19,25,26}$ We also found significant low expression levels of miR-200s miRNAs in clinical RCC specimens in comparison with adjacent normal tissues. However, there was no significant relationship between the miRNA expression and clinicopathological parameters, including tumor stage, tumor grade or patient's prognosis. Our cohort was too small and the follow-up period was too short to evaluate the relationship between them.

EMT is an important step in cancer cell invasion and metastasis and may be triggered by a number of factors, including transforming growth factors. ${ }^{19}$ It has been reported that the miR-200s regulates EMT-activating transcription factors (ZEB1 and ZEB2) and is silenced in mesenchymal-like cancer cells by TGF- $\beta .{ }^{19}$ A recent study demonstrated that the miR-200s members are transactivated by $\mathrm{p} 53$, and p53-regulated miRNAs prevented EMT by targeting ZEB1 and ZEB2 expression. ${ }^{27}$ Thus, the $m i R-200 s$ family, as a new member of the p53 regulatory network, contributes to cancer cell invasion and metastasis by regulating the EMT process in human cancer. 
Table 2 (a) Top 10 highly expressed genes targeted by $m i R-200 \mathrm{~b} / \mathrm{miR}-200 \mathrm{c} / \mathrm{miR}-429$ in clinical $\mathrm{RCCs}(\boldsymbol{P}<0.05)$ and $(\mathrm{b})$ by $m i R-200 \mathrm{al} / \mathrm{miR}$ 141 in clinical RCCs $(P<0.05)$

\begin{tabular}{|c|c|c|c|c|c|}
\hline Entrez gene ID & Symbol & Expression & Fold change & Description & P-value \\
\hline 5744 & PTHLH & Up & 14.53 & Parathyroid hormone-like hormone & \\
\hline 4015 & LOX & Up & 9.98 & Lysyl oxidase & $7.16 \mathrm{E}-08$ \\
\hline 9514 & GAL3ST1 & Up & 6.91 & Galactose-3-O-sulfotransferase 1 & $3.54 \mathrm{E}-07$ \\
\hline 857 & CAV1 & Up & 6.73 & Caveolin 1 , caveolae protein, 22 kDa & $1.18 \mathrm{E}-09$ \\
\hline 6319 & SCD & Up & 6.46 & Stearoyl-CoA desaturase (delta-9-desaturase) & $5.98 \mathrm{E}-09$ \\
\hline 2335 & FN1 & Up & 5.45 & Fibronectin 1 & $2.64 \mathrm{E}-09$ \\
\hline 3778 & KCNMA1 & Up & 4.99 & Potassium large conductance calcium-activated channel, subfamily M, alpha member 1 & $1.47 \mathrm{E}-09$ \\
\hline 170954 & KIAA1949 & Up & 4.82 & Protein phosphatase 1 , regulatory subunit 18 & $4.43 E-09$ \\
\hline 169611 & OLFML2A & Up & 4.79 & Olfactomedin-like 2A & $5.63 \mathrm{E}-07$ \\
\hline 8490 & RGS5 & Up & 4.72 & Regulator of G-protein signaling 5 & $3.69 \mathrm{E}-07$ \\
\hline \multicolumn{6}{|l|}{ (b) } \\
\hline 5744 & PTHLH & Up & 14.53 & Parathyroid hormone-like hormone & $2.84 \mathrm{E}-08$ \\
\hline 9123 & SLC16A3 & Up & 7.6 & Solute carrier family 16 , member 3 (monocarboxylic acid transporter 4 ) & $1.25 \mathrm{E}-09$ \\
\hline 1956 & EGFR & Up & 4.5 & Epidermal growth factor receptor & $1.27 \mathrm{E}-09$ \\
\hline 3759 & KCNJ2 & Up & 3.9 & Potassium inwardly rectifying channel, subfamily J, member 2 & $1.46 \mathrm{E}-09$ \\
\hline 6374 & CXCL5 & Up & 3.89 & Chemokine ( $\mathrm{C}-\mathrm{X}-\mathrm{C}$ motif) ligand 5 & $3.12 \mathrm{E}-03$ \\
\hline 864 & RUNX3 & Up & 3.64 & Runt-related transcription factor 3 & $1.70 \mathrm{E}-08$ \\
\hline 55016 & 1-Mar & Up & 3.09 & Membrane-associated ring finger $(\mathrm{C} 3 \mathrm{HC} 4) 1$, E3 ubiquitin protein ligase & $6.78 \mathrm{E}-08$ \\
\hline 586 & BCAT1 & Up & 3.08 & Branched chain amino-acid transaminase 1 , cytosolic & $4.63 E-08$ \\
\hline 2123 & $\mathrm{EVI} 2 \mathrm{~A}$ & Up & 2.97 & Ecotropic viral integration site $2 \mathrm{~A}$ & $2.94 \mathrm{E}-07$ \\
\hline 639 & PRDM 1 & Up & 2.72 & PR domain containing 1 , with ZNF domain & $3.90 E-09$ \\
\hline
\end{tabular}

Abbreviations: miR, microRNA; RCC, renal cell carcinoma
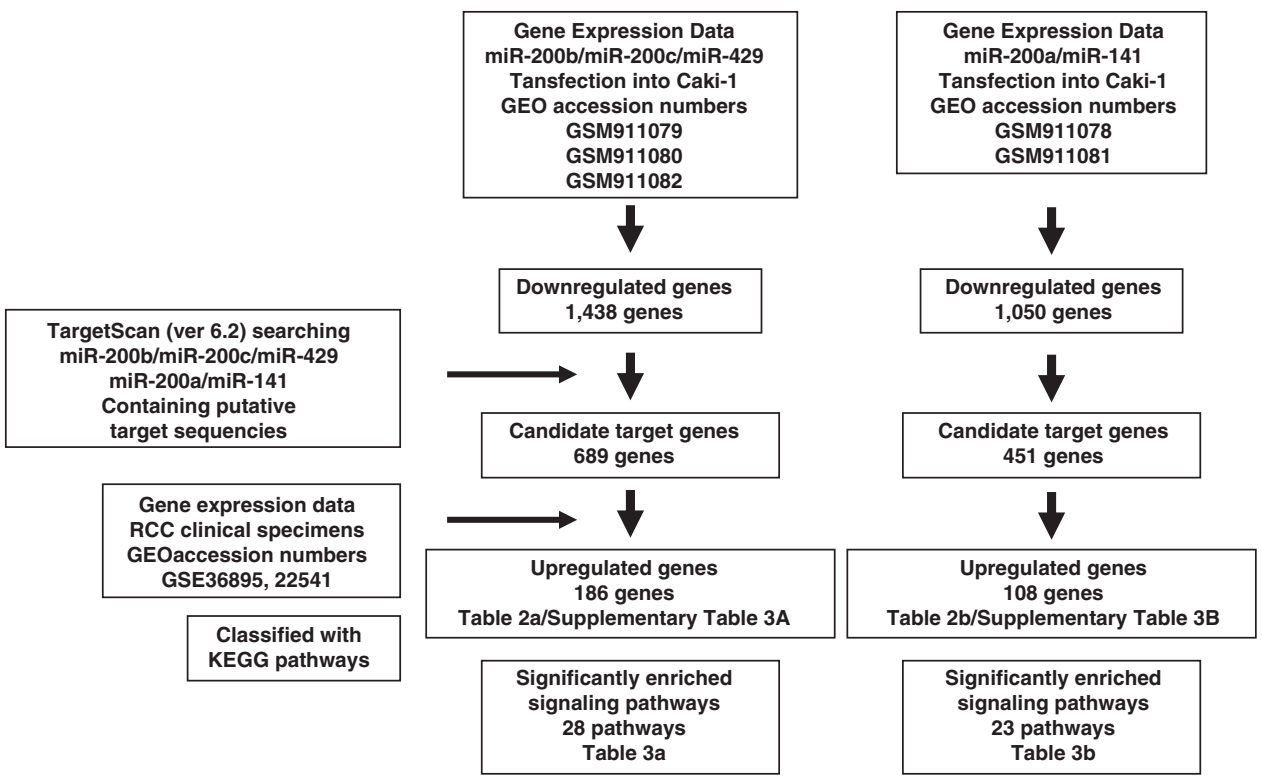

Figure 3 Flow chart of the strategy for analysis of target genes and pathways of miR-200s. Oligo-microarray using miR-200s induced Caki-1 cells showed a total of 1438 downregulated genes in miR-200b/miR-200/miR-429 and 1050 downregulated genes in miR-200a/miR-141 compared with miR-control. Among these genes, 689 and 451 genes had predicted binding sites for miR-200b/miR-200c/miR-429 and miR-200a/miR-141, respectively. We then analyzed the expression levels of these 689 genes for miR-200b/miR-200c/miR-429, and 451 genes for miR-200a/miR-141 between RCCs and normal specimens by using available data sets of GEO database (accession numbers: GSE36895 and GSE22541). The analyses showed that 186 genes for miR-200b/miR-200c/miR-429, and 108 genes for miR-200almiR-141 were significantly upregulated in RCC specimens compared with normal specimens (Tables $2 a$ and $b$ and Supplementary Tables $3 \mathrm{~A}$ and B). These upregulated genes were then analyzed and categorized using the KEGG, and pathways were analyzed using the GENECODIS program. Twenty-eight significantly enriched signaling pathways for miR-200b/miR-200c/miR-429 were shown in Table 3a, and 23 significantly enriched signaling pathways for miR-200a/miR-141 were shown in Table 3b, according to ascending order of $P$-values $(P<0.05)$. 
Table 3 (a) Top 28 enriched pathways downregulated genes by miR-200b/miR-200c/miR-429 in Caki-1 $(P<0.05)$ and (b) top 23 enriched pathways downregulated genes by $m i R-200 a / m i R-141$ in Caki-1 $(P<0.05)$

\begin{tabular}{|c|c|c|c|c|}
\hline $\begin{array}{l}\text { Number } \\
\text { of genes }\end{array}$ & Annotations & $\begin{array}{c}\text { KEGG entry } \\
\text { number }\end{array}$ & P-value & Genes \\
\hline 9 & Focal adhesion & 4510 & $1.60 \mathrm{E}-04$ & ITGA1, ITGA4, LAMC1, COL5A2, CAV1, PAK2, RAPGEF1, CRKL, FN1 \\
\hline 6 & ErbB signaling pathway & 4012 & $4.80 \mathrm{E}-04$ & RPS6KB1, PLCG1, MYC, PAK2, CBL, CRKL \\
\hline 8 & Regulation of actin cytoskeleton & 4810 & $7.80 \mathrm{E}-04$ & PIP4K2A, ITGA1, ITGA4, MSN, PIP4K2B, PAK2, CRKL, FN1 \\
\hline 5 & ECM-receptor interaction & 4512 & $1.91 \mathrm{E}-03$ & ITGA1, ITGA4, LAMC1, COL5A2, FN1 \\
\hline 5 & Small-cell lung cancer & 5222 & $1.91 \mathrm{E}-03$ & $I K B K B, L A M C 1, C D K 2, M Y C, F N 1$ \\
\hline 9 & Pathways in cancer & 5200 & $2.17 \mathrm{E}-03$ & IKBKB, ETS1, LAMC1, PLCG1, CDK2, MYC, CBL, CRKL, FN1 \\
\hline 5 & Fc gamma R-mediated phagocytosis & 4666 & $2.52 \mathrm{E}-03$ & MARCKS, PPAP2B, RPS6KB1, PLCG1, CRKL \\
\hline 4 & Inositol phosphate metabolism & 562 & $3.50 E-03$ & PIP4K2A, PLCB1, PIP4K2B, PLCG1 \\
\hline 5 & Amoebiasis & 5146 & $3.58 \mathrm{E}-03$ & PLCB1, TLR4, LAMC1, COL5A2, FN1 \\
\hline 5 & Neurotrophin signaling pathway & 4722 & $5.87 \mathrm{E}-03$ & IKBKB, MAPK7, PLCG1, RAPGEF1, CRKL \\
\hline 4 & Chronic myeloid leukemia & 5220 & $6.25 E-03$ & $I K B K B, M Y C, C B L, C R K L$ \\
\hline 4 & Renal cell carcinoma & 5211 & $6.30 \mathrm{E}-03$ & ETS1, PAK2, RAPGEF1, CRKL \\
\hline 4 & Bacterial invasion of epithelial cells & 5100 & $6.30 \mathrm{E}-03$ & CAV1, CBL, CRKL, FN1 \\
\hline 5 & Insulin signaling pathway & 4910 & $6.45 E-03$ & IKBKB, RPS6KB1, RAPGEF1, CBL, CRKL \\
\hline 4 & Phosphatidylinositol signaling system & 4070 & $6.75 \mathrm{E}-03$ & PIP4K2A, PLCB1, PIP4K2B, PLCG1 \\
\hline 4 & Salivary secretion & 4970 & $8.98 \mathrm{E}-03$ & KCNMA1, PLCB1, GUCY1A3, GUCY1B3 \\
\hline 4 & Gap junction & 4540 & $9.08 \mathrm{E}-03$ & PLCB1, GUCY1A3, MAPK7, GUCY1B3 \\
\hline 3 & Sphingolipid metabolism & 600 & $9.45 E-03$ & PPAP2B, GAL3ST1, UGCG \\
\hline 4 & T-cell receptor signaling pathway & 4660 & $1.76 \mathrm{E}-02$ & IKBKB, PLCG1, PAK2, CBL \\
\hline 6 & MAPK signaling pathway & 4010 & $1.87 \mathrm{E}-02$ & IKBKB, MAP4K4, MAPK7, MYC, PAK2, CRKL \\
\hline 4 & Vascular smooth muscle contraction & 4270 & $1.94 \mathrm{E}-02$ & KCNMA1, PLCB1, GUCY1A3, GUCY1B3 \\
\hline 3 & Acute myeloid leukemia & 5221 & $2.02 \mathrm{E}-02$ & $I K B K B, R P S 6 K B 1, M Y C$ \\
\hline 4 & Lysosome & 4142 & $2.26 \mathrm{E}-02$ & GM2A, AP1S2, NPC1, CTSO \\
\hline 4 & Toxoplasmosis & 5145 & $2.29 E-02$ & HLA-DMA, IKBKB, TLR4, LAMC1 \\
\hline 2 & Biosynthesis of unsaturated fatty acids & 1040 & $2.57 E-02$ & $S C D, F A D S 1$ \\
\hline 3 & Leishmaniasis & 5140 & $2.59 E-02$ & HLA-DMA, ITGA4, TLR4 \\
\hline 4 & Measles & 5162 & $2.68 \mathrm{E}-02$ & TBK1, MSN, TLR4, CDK2 \\
\hline 3 & Long-term depression & 4730 & $2.72 \mathrm{E}-02$ & PLCB1, GUCY1A3, GUCY1B3 \\
\hline \multicolumn{5}{|l|}{ (b) } \\
\hline 7 & ErbB signaling pathway & 4012 & $1.01 E-06$ & GRB2, ABL2, NCK1, CDKN1A, EGFR, STAT5B, CBL \\
\hline 4 & Chronic myeloid leukemia & 5220 & $3.67 E-03$ & GRB2, CDKN1A, STAT5B, CBL \\
\hline 4 & Gap junction & 4540 & $5.06 \mathrm{E}-03$ & GRB2, GUCY1A3, EGFR, GUCY1B3 \\
\hline 3 & Glioma & 5214 & $1.13 \mathrm{E}-02$ & GRB2, CDKN1A, EGFR \\
\hline 4 & Osteoclast differentiation & 4380 & $1.19 \mathrm{E}-02$ & LILRA1, SQSTM1, GRB2, IFNAR1 \\
\hline 4 & Hepatitis C & 5160 & $1.21 \mathrm{E}-02$ & GRB2, CDKN1A, EGFR, IFNAR1 \\
\hline 4 & Jak-STAT signaling pathway & 4630 & $1.22 \mathrm{E}-02$ & GRB2, STAT5B, CBL, IFNAR1 \\
\hline 6 & Pathways in cancer & 5200 & $1.23 \mathrm{E}-02$ & MSH2, GRB2, CDKN1A, EGFR, STAT5B, CBL \\
\hline 2 & Glycosphingolipid biosynthesis_-ganglio series & 604 & $1.25 \mathrm{E}-02$ & ST3GAL5, ST8SIA1 \\
\hline 3 & Long-term depression & 4730 & $1.31 \mathrm{E}-02$ & GNA13, GUCY1A3, GUCY1B3 \\
\hline 2 & Dorso-ventral axis formation & 4320 & $1.88 \mathrm{E}-02$ & GRB2, EGFR \\
\hline 3 & Prostate cancer & 5215 & $1.97 \mathrm{E}-02$ & GRB2, CDKN1A, EGFR \\
\hline 2 & Glycosaminoglycan biosynthesis-heparan sulfate & 534 & $2.03 E-02$ & HS2ST1, B3GALT6 \\
\hline 4 & Regulation of actin cytoskeleton & 4810 & $2.69 E-02$ & ARPC5, ITGA4, GNA13, EGFR \\
\hline 3 & T-cell receptor signaling pathway & 4660 & $2.74 \mathrm{E}-02$ & GRB2, NCK1, CBL \\
\hline 3 & Vascular smooth muscle contraction & 4270 & $2.99 E-02$ & GNA13, GUCY1A3, GUCY1B3 \\
\hline 2 & Bladder cancer & 5219 & $3.76 \mathrm{E}-02$ & CDKN1A, EGFR \\
\hline 3 & Axon guidance & 4360 & $3.96 \mathrm{E}-02$ & NCK1, NRP1, EPHA2 \\
\hline 4 & Cytokine-cytokine receptor interaction & 4060 & $4.18 \mathrm{E}-02$ & CXCL5, EGFR, ILI8R1, IFNAR1 \\
\hline 2 & Pathogenic Escherichia coli infection & 5130 & 4.77E -02 & ARPC5, NCK1 \\
\hline 2 & Acute myeloid leukemia & 5221 & $4.87 \mathrm{E}-02$ & GRB2, STAT5B \\
\hline 3 & Purine metabolism & 230 & $4.96 \mathrm{E}-02$ & GUCY1A3, GUCY1B3, POLR3GL \\
\hline 1 & Thiamine metabolism & 730 & $4.98 \mathrm{E}-02$ & TPK1 \\
\hline
\end{tabular}

Abbreviations: ECM, extracellular matrix; KEGG, Kyoto Encyclopedia of Genes and Genomes; MAPK, mitogen-activated protein kinase; STAT, signal transducer and activator of transcription. 
We believed that analysis of miR-200s-regulated target genes and pathways could lead to discoveries of new molecular mechanisms in RCC recurrence and metastasis. Accordingly, we utilized the gene expression data from Caki-1 cells transfected with each miR-200s member. In this study, we categorized two groups according to their seed sequences. A total of 186 and 108 genes were listed as candidate oncogenic genes regulated by the $m i R-200 b / m i R-200 c / m i R-429$ and $m i R-200 a / m i R-141$, respectively.

We found the candidate target gene, parathyroid hormone-like hormone $(P T H L H)$, to be the most upregulated in RCC clinical specimens and to have putative $m i R$-200s binding sites in the $3^{\prime}$ untranslated region. $P T H L H$ is a member of the parathyroid hormone family secreted factor expressed in normal tissues and is correlated with a wide range of developmental and physiological processes. ${ }^{28}$ Interestingly, overexpression of PTHLH was reported in several types of cancer, ${ }^{29-33}$ and circulating levels of PTHLH generally correlated with advanced stage of human cancers. ${ }^{32,34-39}$ A recent study demonstrated that PTHLH contributed to breast cancer growth and metastasis through its effects on several crucial signaling molecules; such as, Akt and chemokine receptor. ${ }^{28}$ Thus, the list of genes identified in our screen might be effective for searching oncogenic genes that participate in recurrence and metastasis of RCC.

Furthermore, we categorized these candidate genes into known molecular pathways using KEGG pathways; a total of 28 and 23 pathways were identified as significant $m i R-200 b / m i R-200 c / m i R-429$ and $m i R-200 a / m i R-141$-regulated pathways, respectively. All selected genes were upregulated in RCC clinical specimens by the GEO database. The 'Focal adhesion' and 'ErbB signaling' pathways were the most significantly enriched of the $m i R-200 \mathrm{~b} / \mathrm{miR}-200 \mathrm{c} / \mathrm{miR}$-429-regulated pathways. There were nine genes in the 'Focal adhesion' pathway (ITGA1, ITGA4, LAMC1, COL5A2, CAV1, PAK2, RAPGEF1, CRKL and $F N 1)$ and six genes in the 'ErbB signaling' pathway (RPS6KB1, PLCG1, MYC, PAK2, CBL and CRKL). Among these, fibronectin 1 (FN1), phospholipase C, gamma 1 (PLCG1) and v-myc myelocytomatosis viral oncogene homolog (MYC) have been reported to be directly regulated by $m i R-200 b / m i R-200 c / m i R-429$ in human cancer. ${ }^{40-42}$ The 'ErbB signaling' pathway was also the most significantly enriched pathway in $m i R-200 a / m i R-141$-regulated pathways. There were seven genes identified in this pathway (GRB2, $A B L 2, N C K 1, C D K N 1 A, E G F R, S T A T 5 B$ and $C B L$ ), and signal transducer and activator of transcription $5 \mathrm{~B}$ (STAT5B) have also been reported to be directly regulated by $m i R-200 a{ }^{43}$

As miRNAs are unique in their ability to regulate many protein coding genes, reduced expression of tumor-suppressing miRNAs may cause overexpression of oncogenes in cancer cells. It is very attractive to grasp the tumor-suppressing miRNAs regulated all molecular targets as cancer pathways. Here, we have described pathways mediated by the tumor-suppressing miR-200s members that provide greater insight into RCC and may offer novel therapeutic targets.

In conclusion, EMT-related miR-200s were frequently downregulated in clinical RCC, and they may function as tumor suppressors through targeting several cancer-related genes and pathways. The data from this study may provide a potentially effective and promising strategy for miRNA-associated evidence-based treatment of RCC.

\section{ACKNOWLEDGEMENTS}

This study was supported by the Ministry of Education, Sciences, Sports and Culture, and Grant-in-Aid for Science Research (C), 23501298 and 23592340. We thank Ms Mutsumi Miyazaki for her excellent laboratory assistance.
1 Gupta, K., Miller, J. D., Li, J. Z., Russell, M. W. \& Charbonneau, C. Epidemiologic and socioeconomic burden of metastatic renal cell carcinoma (mRCC): a literature review. Cancer Treat. Rev. 34, 193-205 (2008).

2 Hadoux, J., Vignot, S. \& De La Motte Rouge, T. Renal cell carcinoma: focus on safety and efficacy of temsirolimus. Clin. Med. Insights Oncol. 4, 143-154 (2010).

3 Margulis, V., Master, V. A., Cost, N. G., Leibovich, B. C., Joniau, S., Kuczyk, M. et al. International consultation on urologic diseases and the European Association of Urology international consultation on locally advanced renal cell carcinoma. Eur. Urol. 60, 673-683 (2011).

4 Carthew, R. W. \& Sontheimer, E. J. Origins and mechanisms of miRNAs and siRNAs. Cell 136, 642-655 (2009).

5 Bartel, D. P. MicroRNAs: genomics, biogenesis, mechanism, and function. Cell 116, 281-297 (2004).

6 Calin, G. A. \& Croce, C. M. MicroRNA signature in human cancers. Nat. Rev. Cancer 6, 857-866 (2006)

7 Wu, X., Weng, L., Li, X., Guo, C., Pal, S. K., Jin, J. M. et al. Identification of a 4-microRNA signature for clear cell renal cell carcinoma metastasis and prognosis. PLoS One 7, e35661 (2012).

8 Hidaka, H., Seki, N., Yoshino, H., Yamasaki, T., Yamada, Y., Nohata, N. et al. Tumor suppressive microRNA-1285 regulates novel molecular targets: aberrant expression and functional significance in renal cell carcinoma. Oncotarget 3, 44-57 (2012).

9 Wulfken, L. M., Moritz, R., Ohlmann, C., Holdenrieder, S., Jung, V., Becker, F. et al. MicroRNAs in renal cell carcinoma: diagnostic implications of serum miR-1233 levels. PLoS One 6, e25787 (2011).

10 White, N. M., Bao, T. T., Grigull, J., Youssef, Y. M., Girgis, A., Diamandis, M. et al. miRNA profiling for clear cell renal cell carcinoma: biomarker discovery and identification of potential controls and consequences of miRNA dysregulation. J. Urol. 186, 1077-1083 (2011).

11 Weng, L., Wu, X., Gao, H., Mu, B., Li, X., Wang, J. H. et al. MicroRNA profiling of clear cell renal cell carcinoma by whole-genome small RNA deep sequencing of paired frozen and formalin-fixed, paraffin-embedded tissue specimens. J. Pathol. 222, 41-51 (2010).

12 Liu, H., Brannon, A. R., Reddy, A. R., Alexe, G., Seiler, M. W., Arreola, A. et al. Identifying mRNA targets of microRNA dysregulated in cancer: with application to clear cell renal cell carcinoma. BMC Syst. Biol. 4, 51 (2010).

13 Juan, D., Alexe, G., Antes, T., Liu, H., Madabhushi, A., Delisi, C. et al. Identification of a microRNA panel for clear-cell kidney cancer. Urology 75, 835-841 (2010).

14 Chow, T. F., Youssef, Y. M., Lianidou, E., Romaschin, A. D., Honey, R. J., Stewart, R. et al. Differential expression profiling of microRNAs and their potential involvement in renal cell carcinoma pathogenesis. Clin. Biochem. 43, 150-158 (2010).

15 Jung, M., Mollenkopf, H. J., Grimm, C., Wagner, I., Albrecht, M., Waller, T. et al. MicroRNA profiling of clear cell renal cell cancer identifies a robust signature to define renal malignancy. J. Cell. Mol. Med. 13, 3918-3928 (2009).

16 Nakada, C., Matsuura, K., Tsukamoto, Y., Tanigawa, M., Yoshimoto, T., Narimatsu, T. et al. Genome-wide microRNA expression profiling in renal cell carcinoma: significant down-regulation of miR-141 and miR-200c. J. Pathol. 216, 418-427 (2008).

17 Huber, M. A., Kraut, N. \& Beug, H. Molecular requirements for epithelial-mesenchymal transition during tumor progression. Curr. Opin. Cell. Biol. 17, 548-558 (2005).

$18 \mathrm{Xu}$, J., Lamouille, S. \& Derynck, R. TGF-beta-induced epithelial to mesenchymal transition. Cell Res. 19, 156-172 (2009).

19 Gregory, P. A., Bert, A. G., Paterson, E. L., Barry, S. C., Tsykin, A., Farshid, G. et al. The miR-200 family and miR-205 regulate epithelial to mesenchymal transition by targeting ZEB1 and SIP1. Nat. Cell. Biol. 10, 593-601 (2008).

20 Sobin, L. H., Gospodarowicz, M. K. \& Wittenkind, C. TNM Classification of Malignant Tumours, 7th ed. International Union Against Cancer 255-257 (Wiley-Liss, New York, NY, USA, 2009).

21 Nohata, N., Hanazawa, T., Kikkawa, N., Mutallip, M., Fujimaru, L., Yoshino, H. et al. Caveolin-1 mediates tumor cell migration and invasion and its regulation by miR-133a in head and neck squamous cell carcinoma. Int. J. Oncol. 38, 209-217 (2011).

22 Kawakami, K., Enokida, H., Chiyomaru, T., Tatarano, S., Yoshino, H., Kagara, I. et al. The functional significance of MiR-1 and miR-133a in renal cell carcinoma. Eur. J. Cancer 48, 827-836 (2012).

23 Sugimoto, T., Seki, N., Shimizu, S., Kikkawa, N., Tsukada, J., Shimada, H. et al. The galanin signaling cascade is a candidate pathway regulating oncogenesis in human squamous cell carcinoma. Genes Chromosomes Cancer 48, 132-142 (2009).

24 Girgis, A. H., lakovlev, V. V., Beheshti, B., Bayani, J., Squire, J. A., Bui, A. et al. Multilevel whole-genome analysis reveals candidate biomarkers in clear cell renal cell carcinoma. Cancer Res. 72, 5273-5284 (2012).

25 Ladeiro, Y., Couchy, G., Balabaud, C., Bioulac-Sage, P., Pelletier, L. \& Rebouissou, S. MicroRNA profiling in hepatocellular tumors is associated with clinical features and oncogene/tumor suppressor gene mutations. Hepatology 47, 1955-1963 (2008).

26 Dahiya, N., Sherman-Baust, C. A., Wang, T. L., Davidson, B., Shih, I. e. M. \& Zhang, Y. MicroRNA expression and identification of putative miRNA targets in ovarian cancer. PLoS One 18, e2436 (2008).

27 Kim, T., Veronese, A., Pichiorri, F., Lee, T. J., Jeon, Y. J., Volinia, S. et al. P53 regulates epithelial-mesenchymal transition through microRNAs targeting ZEB1 and ZEB2. J. Exp. Med. 208, 875-883 (2011).

$28 \mathrm{Li}$, J., Karaplis, A. C., Huang, D. C., Siegel, P. M., Camirand, A. \& Yang, X. F. PTHrP drives breast tumor initiation, progression, and metastasis in mice and is a potential therapy target. J. Clin. Invest. 121, 4655-4669 (2011). 
29 Nishigaki, Y., Ohsaki, Y., Toyoshima, E. \& Kikuchi, K. Increased serum and urinary levels of a parathyroid hormone-related protein $\mathrm{COOH}$ terminus in non-small cell lung cancer patients. Clin. Cancer Res. 5, 1473-1481 (1995).

30 Dougherty, K. M., Blomme, E. A., Koh, A. J., Henderson, J. E., Pienta, K. J., Rosol, T. J. et al. Parathyroid hormone-related protein as a growth regulator of prostate carcinoma. Cancer Res. 59, 6015-6022 (1999).

31 Agouni, A., Sourbier, C., Danilin, S., Rothhut, S., Lindner, V., Jacqmin, D. et al. Parathyroid hormone-related protein induces cell survival in human renal cell carcinoma through the PI3K Akt pathway: evidence for a critical role for integrin-linked kinase and nuclear factor kappa B. Carcinogenesis 28, 1893-1901 (2007).

32 Nishihara, M., Ito, M., Tomioka, T., Ohtsuru, A., Taguchi, T. \& Kanematsu, T. Clinicopathological implications of parathyroid hormone-related protein in human colorectal tumours. J. Pathol. 187, 217-222 (1999).

33 Alipov, G. K., Ito, M., Nakashima, M., Ikeda, Y., Nakayama, T., Ohtsuru, A. et al. Expression of parathyroid hormone-related peptide (PTHrP) in gastric tumours. J. Pathol. 182, 174-179 (1997).

34 Deftos, L. J., Barken, I., Burton, D. W., Hoffman, R. M. \& Geller, J. Direct evidence that PTHrP expression promotes prostate cancer progression in bone. Biochem. Biophys. Res. Commun. 327, 468-472 (2005).

35 Firkin, F., Seymour, J. F., Watson, A. M., Grill, V. \& Martin, T. J. Parathyroid hormonerelated protein in hypercalcaemia associated with haematological malignancy. $\mathrm{Br}$. J. Haematol. 94, 486-492 (1996).
36 Kissin, M. W., Henderson, M. A., Danks, J. A., Hayman, J. A., Bennett, R. C. \& Martin T. J. Parathyroid hormone related protein in breast cancers of widely varying prognosis. Eur. J. Surg. Oncol. 19, 134-142 (1993).

37 Kremer, R., Shustik, C., Tabak, T., Papavasiliou, V. \& Goltzman, D. Parathyroid hormone-related peptide in hematologic malignancies. Am. J. Med. 100, 406-411 (1996).

38 Rankin, W., Grill, V. \& Martin, T. J. Parathyroid hormone-related protein and hypercalcemia. Cancer 80, 1564-1571 (1997).

39 Truong, N. U., de B Edwardes, M. D., Papavasiliou, V., Goltzman, D. \& Kremer, R Parathyroid hormone-related peptide and survival of patients with cancer and hypercalcemia. Am. J. Med. 115, 115-121 (2003).

40 Howe, E. N., Cochrane, D. R. \& Richer, J. K. Targets of miR-200c mediate suppression of cell motility and anoikis resistance. Breast Cancer Res. 13, R45 (2011).

41 Uhlmann, S., Zhang, J. D., Schwäger, A., Mannsperger, H., Riazalhosseini, Y. Burmester, S. et al. miR-200bc/429 cluster targets PLCgamma1 and differentially regulates proliferation and EGF-driven invasion than miR-200a/141 in breast cancer. Oncogene 29, 4297-4306 (2010).

42 Sun, T., Wang, C., Xing, J. \& Wu, D. miR-429 modulates the expression of c-myc in human gastric carcinoma cells. Eur. J. Cancer 47, 2552-2559 (2011).

43 Williams, K. C., Renthal, N. E., Condon, J. C., Gerard, R. D. \& Mendelson, C. R MicroRNA-200a serves a key role in the decline of progesterone receptor function leading to term and preterm labor. Proc. Natl. Acad. Sci. USA 109, 7529-7534 (2012)

Supplementary Information accompanies the paper on Journal of Human Genetics website (http://www.nature.com/jhg) 